\title{
The analysis of phytoplankton diversity in water among Xiaowutai
}

\section{Mountain Basin (China)}

\author{
Hong-wei WANG ${ }^{1,2, a}$, Lin-feng $\mathrm{LI}^{1, \mathrm{~b}}$, Chun-long $Z \mathrm{HAO}^{3, \mathrm{c}^{*}}$, Rong ZHENG ${ }^{4, \mathrm{~d}}$, \\ Zhong FU ${ }^{5, e}$,Ni MING ${ }^{1, f}$, Pei-pei WANG ${ }^{1, g}$, Shao-ying XING ${ }^{1, h}$ Shuo CHANG $^{1, \mathrm{i}}$, \\ Min-zhe ZHANG ${ }^{6, j}$, Xiao-dan LIN ${ }^{1, K}$ \\ 1.The Key Laboratory of Zoological Systematics and Application, College of Life Sciences, Hebei \\ University, Baoding, Hebei, P.R. China \\ 2.Laboratory of Environmental Toxicology and Aquatic Ecology, Ghent University, Ghent, Belgium \\ 3.Marine Living Resources and Environment Key Laboratory of Hebei Province, Qinhuangdao, \\ P.R .China \\ 4.Institute of Supervision and Inspection of Quality and Technology, Shanghai, P.R .China \\ 5.Hebei Ocean \& Fishery Sciences Research Institute, Qinhuangdao, P.R .China \\ 6.School of computer science and technology, Hebei University, Baoding, P.R. China

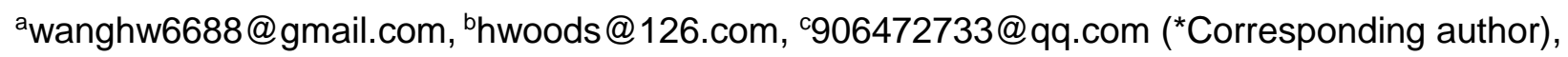 \\ d13323249639@163.com, ehydr6688@126.com, '2891285682@qq.com, \\ gok200090@hotmail.com, ${ }^{\mathrm{h}} 13722283189 @ 126 . c o m$,
}

i511898215@qq.com,'hydro6688@163.com, ${ }^{\mathrm{K}}$ bio2008@126.com

Key words: Xiaowutai Mountain; waters; phytoplankton; diversity

Abstract. Based on the investigation of phytoplankton in 14 samples of the waters among Xiaowutai Mountain in August 2016, the species composition, evenness, abundance and diversity of phytoplankton in the Xiaowutai Mountain were studied, and then evaluated the quality conditions of water. The results showed that the samples collected from the phytoplankton survey in the Xiaowutai Mountain were identified by microscopic observation. There were 108 species of 8 phyla, among which the dominant species of cyanobacteria species accounted for 41 species, accounting for $37.96 \%$ of all species, followed by diatoms and green algae. Dominant species are mainly Microcystis flos-aquae, Anbaena circinalis, Melosira sp., Oedogonium sp., Closterium sp. There is unique geographical environment among Xiaowutai Mountain. It is a national nature reserve and is rated as the national AA level tourist scenic spot. The ecological types of phytoplankton are varied. The Diversity index was 0.687 - 4.905. The degree of evenness was $0.226-1.637$ and the richness were 2.389 -8.564.4. The diversity evenness and richness index were fluctuated in August (the wet season). The results show that most of the sampling points are not highly nutritious and the water quality is good.

\section{Introduction}

Xiaowutai Mountain, located in Yuxian and Zhuolu two counties, Zhangjiakou area, in the northwest of Hebei Province. It is a national nature reserve and has abundant water resources. Phytoplankton is the basic link between the primary producers and the food chain in the ecosystems, 
and the response to environmental changes is very rapid ${ }^{[1]}$. The phytoplankton community structure, biomass and intensity and frequency of occurrence of bloom all can be used as an important indicator of long-term changes in these water ecosystems ${ }^{[2]}$. This study provides the scientific evidence to promote the sustainable development of water and ecological environment protection.

\section{Materials and methods}

\section{Sampling sites}

The sampling site was located in Zhangjiakou Xiaowutai Nature Reserve. The experimental group sampled a total of 14 samples in the waters of Yuxian Jinhekou Scenic Spot in Hebei Province, detection of the sampling point of water quality, the specific situation in Table 1 for hydrological physical and chemical factor statistics.

Table 1 The statistics of hydrological physical and chemical factor in sites

\begin{tabular}{|c|c|c|c|c|c|c|c|c|c|}
\hline sample & $\begin{array}{r}\text { Dissol } \\
\text { oxygen } \\
\text { (DO }\end{array}$ & $\mathrm{pH}$ & TDS & DO\% & ORP & $\begin{array}{l}\text { Total } \\
\text { phosphorus }\end{array}$ & $\begin{array}{l}\text { Ammonia } \\
\text { nitrogen }\end{array}$ & $\begin{array}{l}\text { Total } \\
\text { nitrogen }\end{array}$ & COD \\
\hline 1 & 8.67 & 8.78 & 0.2 & 89.1 & 269 & 0.09 & 1.44 & 2.18 & 30.3 \\
\hline 2 & 8.97 & 8.43 & 0.2 & 85.5 & 255 & 0.03 & 1.33 & 2.48 & $<15$ \\
\hline 3 & 9.52 & 8.48 & 0.2 & 89.6 & 262 & 0.02 & 1.06 & 2.22 & 35.3 \\
\hline 4 & 9.01 & 8.76 & 0.2 & 90.1 & 246 & 0.02 & 0.61 & 2.08 & 16.8 \\
\hline 5 & 9.10 & 8.61 & 0.2 & 92.4 & 269 & 0.06 & 0.97 & 2.65 & 32.4 \\
\hline 6 & 8.62 & 8.68 & 0.2 & 87.4 & 257 & 0.07 & 0.56 & 2.18 & 31.1 \\
\hline 7 & 8.05 & 7.90 & 0.3 & 77.5 & 253 & 0.07 & 0.09 & 2.16 & $<15$ \\
\hline 8 & 8.16 & 8.83 & 0.3 & 78.8 & 260 & 0.10 & 0.18 & 2.61 & 34.3 \\
\hline 9 & 9.51 & 9.44 & 0.3 & 97.2 & 246 & 0.08 & 0.08 & 2.5 & 36.2 \\
\hline 10 & 9.68 & 9.55 & 0.3 & 95.2 & 261 & 0.07 & 0.48 & 2.09 & 45.7 \\
\hline 11 & 17.3 & 10.97 & 0.6 & 94.6 & 269 & 0.06 & 0.57 & 7.60 & 24.1 \\
\hline 12 & 9.37 & 9.69 & 0.2 & 87.2 & 252 & 0.04 & 0.87 & 2.39 & 33.9 \\
\hline 13 & 8.26 & 7.65 & 0.2 & 80.6 & 213 & 0.35 & 0.44 & 2.25 & $<15$ \\
\hline 14 & 9.11 & 8.58 & 0.2 & 82.8 & 209 & 0.18 & 0.53 & 2.04 & $<15$ \\
\hline
\end{tabular}

\section{The research methods}

Phytoplankton functional group is the starting point of the morphological, physiological and ecological characteristics of phytoplankton similar to a group, within the group have the same or similar survival strategy (niche), each group can reflect the specific habitat type ${ }^{[3]}$.

\section{Results}

After 14 phytoplankton samples survey, in the Xiaowutai mountain water of the communist party of China identified 109 species and varieties of phytoplankton, belonging to 8 phyla. Xiaowutai mountain water phytoplankton classification is shown in Figure 1. 


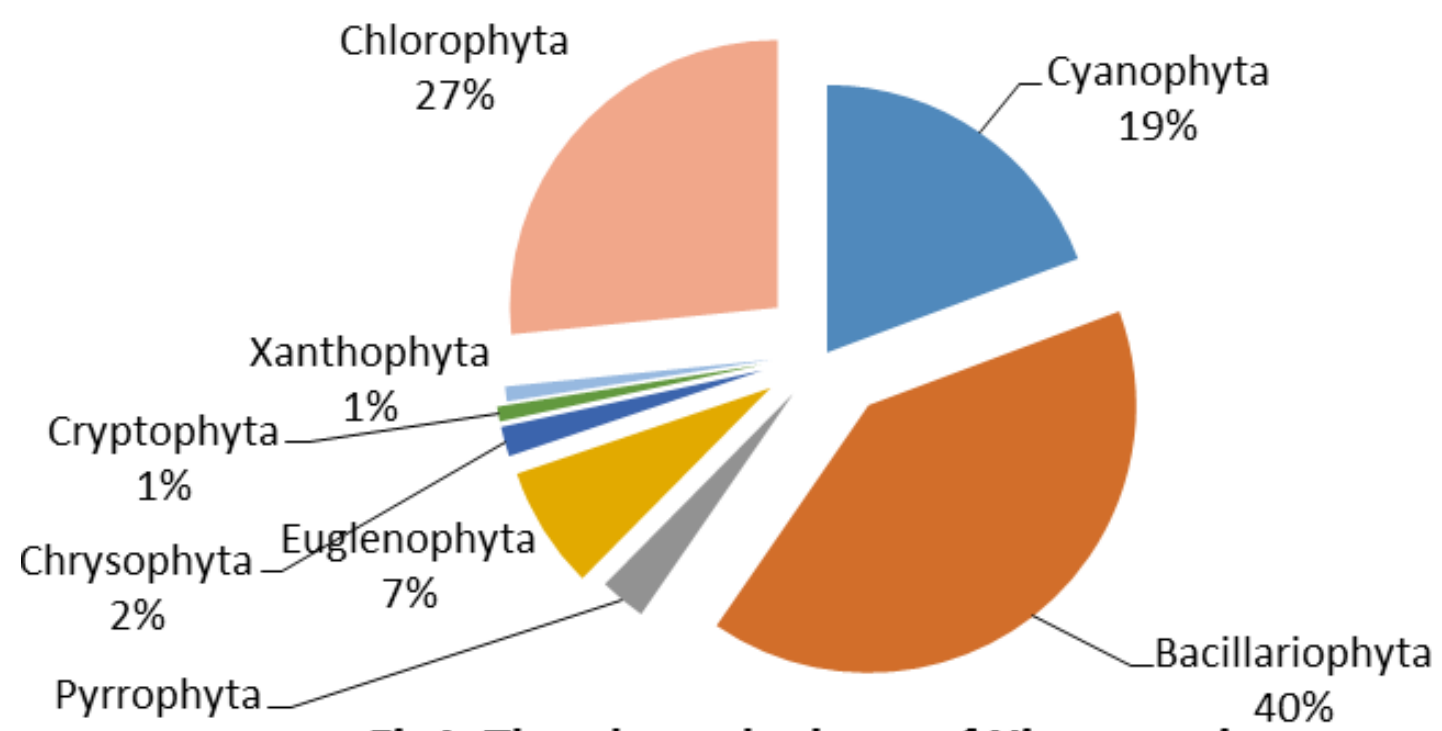

$3 \%$

Fig1 The phytoplankton of Xiao wutai

According to the statistical results of Shannon-Wiener diversity index, evenness index and richness index of phytoplankton at each sampling point in the Jinhe River, Xiaowutai Mountain. In summary, the diversity of phytoplankton in the Jinsha Watershed is very good, and it is suitable for the growth of various phytoplanktons.

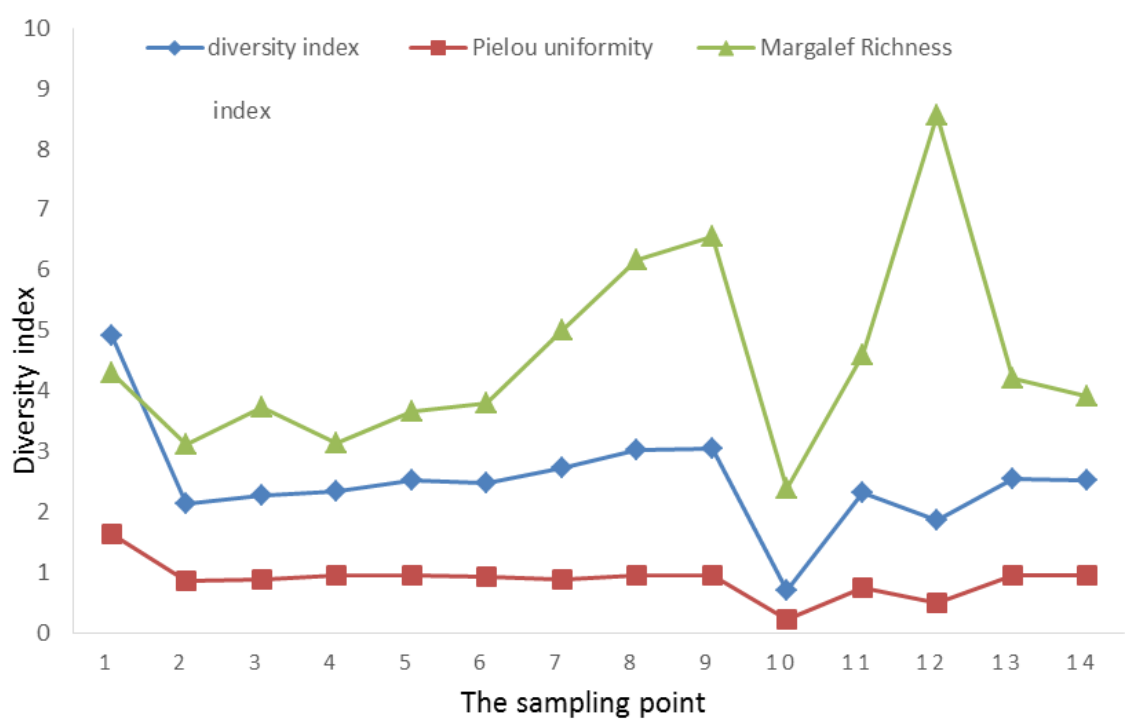

Fig.2 The phytoplankton diversity index of each sample point of xiaowutai water area

\section{Conclusion}

Phytoplankton classification method is to reveal the phytoplankton community succession law, to carry out freshwater ecological research work foundation and important tools ${ }^{[4]}$. A total of 109 species of phytoplankton were observed during the investigation of the Xiaowutai Mountain. The community composition was dominated by diatomophytes and chlorophyta. The dominant species were mainly Anabaena sp., Microcystis Algae, Crescent Algae.

From the overall river basin, the biodiversity index of sampling points 7-9 is higher than that of other places, the pollution of water is less, the biodiversity index of No. 10 is obviously lower than 
other places, and the eutrophication of water is serious. On the whole, the species diversity of phytoplankton in the Xiaowutai Mountain is relatively high, while the Shannon-Wiener index is between the moderate pollution and the mild pollution, and the species richness is relatively low. Therefore, the use of biodiversity index to assess the level of water quality non-nutrient, its accuracy is still has certain limitation, in the future research needs to be further ${ }^{[5]}$.

\section{Acknowledgements}

This research was supported by the Subject of advantage characteristic biology in Hebei Province; The natural science foundation of Hebei Province（D2013201105）; Students studying abroad preferred activities of science and technology project funding projects in hebei province (C2015003034) ; The Project-sponsored by SRF for ROCS, SEM(2014); Hydrobiology key discipline in Hebei province; Modern Agriculture Industry Technology System Characteristic Seafood Innovation Team in Hebei Province (2013-2017); Projects of coastal habitat restoration in Qinhuangdao (Provincial Science and Technology Department, No. 15273303D) ; Hebei Provincial Key Laboratory of operating subsidy (item number: 14967611D); National Innovation Innovation Training Program, Hebei University (201610075014); Graduate Student Innovation Fund Project, Hebei University (X2016072); Field Innovation Entrepreneurship Training Program, Hebei University (2016084); Laboratory Open Project, Hebei University (sy201662).

\section{Reference}

[1] Reynolds C. Variability in the provision and function of mucilage in phytoplankton: facultative responses to the environment. Hydrobiologia, 2007, 578 (1):37 -45.

[2] Cellamare M,Morin S,Coste $\mathrm{M}$ et al.Ecological assessment of French Atlantic lakes based on phytoplankton, phytobenthos and macrophytes. Environmental Monitoring and Assessment, 2012, 184 (8):4685-4708.

[3] Hu Ren, blue in qian, XiaoLiJuan, bo-ping han. Freshwater phytoplankton concept of functional groups, the method and application [J]. Journal of lake science, 2015, (01): 11-23.

[4] Ellen pau, Zhu Jin yong, open macro, lu Wan Li, Mao Xiaohua. Freshwater phytoplankton functional category classification is put forward, the development and application [J]. Journal of applied ecology, 2014, (6) : 1833-1840.

[5] gui-sen du, wang jian hall. Miyun reservoir of phytoplankton community structure and density [J]. Journal of plant ecology, 2001, 25 (4): 501-504. 